\title{
Descrição do ciclo reprodutivo de Donax hanleyanus (Bivalvia, Donacidae) no sul do Brasil
}

\author{
Guacira M. Gil \& José W. Thomé
}

Laboratório de Malacologia, Pontifícia Universidade Católica do Rio Grande do Sul, Av. Ipiranga, 6681, prédio 12-D, sala 340, 90619-900, Porto Alegre, RS, Brasil. (guagil@ pop.com.br)

\begin{abstract}
Description of the reproductive cycle of Donax hanleyanus (Bivalvia, Donacidae) in southern Brazil. The changes in gonadal development in male and female of Donax hanleyanus Philippi, 1847 are described. Gonad development and spawning seasons were assessed by monthly sampling of population from Arroio Teixeira beach, southern Brazil. Histological examination of clams showed in the most of the adult population sampled that the spawning is practically continuous, without any period of sexual rest. There are two main periods of gametogenesis, in winter and in summer, being the degree of maturation longer in the latter. The water temperature is stimulant on gametes proliferation and spawning.
\end{abstract}

KEYWORDS. Donax, reproductive cycle, spawning season.

\section{INTRODUÇÃO}

Donax hanleyanus Philippi, 1847 é um bivalve abundante na zona de varrido das praias arenosas do Rio Grande do Sul, Brasil. Segundo Cardoso \& Veloso (2003), sua distribuição se estende de $17^{\circ} \mathrm{S}$ (região tropical) até $37^{\circ} \mathrm{S}$ (região temperada). A diversidade e a ampla distribuição do gênero Donax Linné, 1758 (MORRISON, 1971; ANSELL, 1983; SiCARDI, 1993), as grandes flutuações na densidade populacional (COE, 1956; Penchaszadeh \& Olivier, 1975; Defeo \& Alava, 1995) e a importância ecológica desses animais (McLACHLAN \& HaneKon, 1979; McLachlan et al., 1981; Cardoso \& VeLoso, 2003), associadas à sua importância econômica (McLachlan et al., 1996), têm gerado uma série de investigações sobre a ecologia e a biologia desses moluscos.

A reprodução de $D$. hanleyanus foi estudada por Penchaszadeh \& Olivier (1975), para populações argentinas. No entanto, os autores examinaram histologicamente apenas espécimes com comprimento de concha superior a $20 \mathrm{~mm}$.

O objetivo é descrever o ciclo gametogênico de $D$. hanleyanus na costa sul do Brasil, relacionando-se os resultados às variações sazonais de temperatura e salinidade.

\section{MATERIAL E MÉTODOS}

A investigação foi realizada na praia de Arroio Teixeira (29³8'44"S; 4956'49' W), localizada no litoral norte do Rio Grande do Sul. Fisicamente, pode ser caracterizada como uma praia arenosa exposta, constituída por dunas frontais e uma larga faixa (média de $66 \mathrm{~m}$ ) de areia fina. Apresenta pequena declividade $(2,33 \%)$ e variação média da maré de $0,5 \mathrm{~m}$, sendo classificada morfodinamicamente como uma praia dissipativa $(\Omega=7,6)$ (GiL \& Thоме́, 2001).

As amostragens foram realizadas durante 15 meses consecutivos, entre outubro de 2001 e dezembro de 2002, tendo sido registradas a salinidade e a temperatura da água. As amostras foram obtidas em três transectos, dispostos em ângulo reto à linha de praia, utilizando-se um amostrador cilíndrico de $10 \mathrm{~cm}$ de altura e $25 \mathrm{~cm}$ de diâmetro. O sedimento e o material biológico foram passados por uma peneira de malha de $2 \mathrm{~mm}$ e os exemplares animais fixados com formaldeído $4 \%$, imediatamente após a coleta, como forma de minimizar modificações fisiológicas ou postmorten (MesQuiTa et al., 2001). A fixação se deu com os animais ainda no interior da concha, porém com as valvas entreabertas.

Para a avaliação do ciclo reprodutivo, a cada mês, as gônadas de aproximadamente 20 indivíduos foram submetidas a exame histológico. O comprimento dos espécimes variou de 7,0 $\mathrm{mm}$ a $28,3 \mathrm{~mm}$, sendo excluídos da análise apenas os indivíduos muito jovens da população. Após a retirada das valvas, foi seccionada a porção imediatamente posterior aos palpos labiais, incluindo gônadas e glândula digestiva. As peças passaram por processo de desidratação, diafanização, inclusão em parafina e foram seccionadas em cortes transversais, sagitais e longitudinais, de $7 \mu \mathrm{m}$ de espessura, sendo coradas com hematoxilina-eosina, seguindo procedimento padrão (VÉLEZ, 1985; BORZONE $e t$ al., 2001). Os cortes foram analisados em microscópio de luz, acoplado com câmera digital para obtenção de imagens.

A fim de verificar o grau de correlação entre o ciclo reprodutivo e algumas variáveis ambientais, estabeleceuse a regressão linear entre os valores de temperatura e de salinidade mensais, e o número de indivíduos adultos, em cada um dos estágios reprodutivos.

\section{RESULTADOS}

Em D. hanleyanus não é possível distinguir o sexo ou o estágio de maturação apenas pela observação externa das gônadas. Macroscopicamente, as partes moles apresentam-se com coloração esbranquiçada uniforme, sem variação perceptível a olho nu, exceção feita à 
glândula digestiva, cuja coloração varia de verde a marrom-escura, dependendo do alimento ingerido pelo animal.

Durante o processo de dissecação, alguns indivíduos apresentam aumento de volume na região gonadal, posteriormente confirmado como sendo decorrente de um avançado estágio de maturação. No entanto, não se pode considerar apenas este aspecto para determinação do período reprodutivo, uma vez que em muitos indivíduos onde este aumento de volume não era evidente, a análise histológica mostrou que eram indivíduos totalmente maduros ou em diferentes estágios de maturação.

As gônadas de $D$. hanleyanus são pares e, em ambos os sexos, apresentam coloração amareloesbranquiçada, estando situadas na massa visceral, na região dorso-lateral do corpo, circundando a glândula digestiva. Envolvem, também, o estômago, o saco do estilete cristalino e o intestino, não havendo a penetração de tecido gonadal na glândula digestiva. Essas características tornam difícil o isolamento e extração somente das gônadas.

De um total de 347 indivíduos analisados histologicamente, 27 foram classificados como indeterminados, ou seja, indivíduos nos quais ainda não era possível a determinação do sexo. Dos 320 indivíduos adultos, 151 eram fêmeas e 169, machos. Não foi registrado hermafroditismo na população, comprovando-se que a espécie é dióica.

Após uma análise preliminar dos cortes histológicos, foram definidos quatro estágios de maturidade sexual para cada sexo, adaptando-se à espécie em estudo, as escalas propostas por NARCHI (1976) e BORZONE et al. (2001).

A condição de maturação de cada espécime foi definida a partir da predominância de um dos estágios, já que os folículos gonadais de D. hanleyanus podem apresentar diferentes estágios de maturação.

Início da maturação nos machos. Estágio caracterizado pelo início do desenvolvimento gonadal nos jovens ou a retomada do ciclo reprodutivo nos adultos. Apresenta muitas células imaturas, de tamanho pequeno e uniforme, com material cromático fortemente corado. As espermatogônias estão bem visíveis junto à parede dos folículos, que possuem diâmetro médio de 35 mm. Ainda não há uma série espermática definida (fig. 1). Continuando o processo de espermatogênese, as espermatogônias crescem, mas permanecem dispostas junto à parede do folículo. Sucessivas divisões originam a série espermática radial completa, ainda que esta não esteja muito clara. Durante o processo, as paredes dos folículos se expandem e estes podem apresentar diâmetro de até $380 \mu \mathrm{m}$.

Maturação máxima. Estágio identificado pela presença de série espermática radial bem desenvolvida. Nesta fase, na maioria dos folículos gonadais, há o predomínio de espermátides e espermatozóides completamente maduros, ocupando a região central do lúmen. Espermatogônias e espermatócitos ainda são numerosos. Alguns folículos exibem um começo de emissão, reconhecível pela reorganização dos espermatozóides, dando um aspecto de vórtice ao lúmen folicular (fig. 2).
Emissão parcial com proliferação. Fase onde ocorre a emissão parcial de espermatozóides, mas os elementos proliferativos continuam sendo formados, indicando um ciclo contínuo de produção de gametas. Observam-se muitas espermatogônias próximas à parede folicular. Muitos espermatozóides ainda permanecem no lúmen e são liberados progressivamente, até o esvaziamento quase total do folículo (fig. 3).

Emissão total. Estágio caracterizado pela existência de folículos com paredes de formato irregular, contendo algumas células maduras residuais, no lúmen, e outras, células iniciais da série espermatogênica, desprendidas da parede folicular, marcando o final da gametogênese. Em diferentes regiões das gônadas de um mesmo animal, pode-se constatar a reabsorção dos gametas residuais em alguns folículos e, em outros, o espessamento da parede, fato indicativo da retomada do ciclo reprodutivo (fig. 4).

Início da maturação nas fêmeas. Estágio muito semelhante àquele dos machos. Os folículos são de diâmetros reduzidos (35 $\mu \mathrm{m}$, em média), apresentando células proliferativas (oogônias), de tamanho pequeno e uniforme (média de 7,5 x 12,5 $\mu \mathrm{m}$ ), fortemente coradas. Tais células encontram-se, sempre, aderidas à parede folicular (fig. 5). As oogônias crescem, com aumento simultâneo do núcleo e do nucléolo, atingindo diâmetro médio de $20 \mu \mathrm{m}$, quando iniciam sua divisão. Nesta fase da oogênese podem-se observar células em diferentes estágios proliferativos, no interior do folículo: oogônias primárias, oogônias secundárias, oócitos primários, oócitos em fase pré-vitelogênica e oócitos em vitelogênese. Nesta fase, em cada corte transversal do folículo pode-se contar entre 7 e 18 células em diferentes estágios de desenvolvimento.

Maturação máxima. Estágio em que os folículos estão expandidos, com diâmetro médio de $200 \mu \mathrm{m}$, podendo atingir $250 \mu \mathrm{m}$. Nos cortes transversais dos folículos pode-se observar o seccionamento de aproximadamente quatro óvulos maduros por folículo. Muitos óvulos apresentam formato poliédrico, devido à compressão no interior do folículo. Em cortes longitudinais, observa-se que os oócitos em vitelogênese apresentam forma elíptica, estando ligados à parede folicular por um pedúnculo. Nesta fase, núcleo e nucléolo estão bastante desenvolvidos e, em uma célula de 67 x $42 \mu \mathrm{m}$, o núcleo pode atingir $25 \mu \mathrm{m}$ e o nucléolo $10 \mu \mathrm{m}$ (fig. 6).

Desova parcial com proliferação. Estágio do ciclo reprodutivo onde se verifica a ocorrência de desova juntamente com a proliferação de oócitos, evidenciando que, assim como nos machos, a produção de gametas e a desova são contínuas. Em cortes transversais, os folículos mantêm a forma esférica e sua parede torna-se mais espessa. Os animais ainda apresentam células maduras e íntegras. Ao mesmo tempo, podem ser observados muitos elementos proliferativos (oócitos), de tamanhos variados, presos à parede dos folículos (fig. 7).

Desova total. Nesta fase, praticamente todos os óvulos maduros já foram liberados, levando ao esgotamento da maioria dos folículos gonadais. Os óvulos residuais começam a ser reabsorvidos, perdendo sua forma esférica. Os folículos contraem-se, perdem sua 


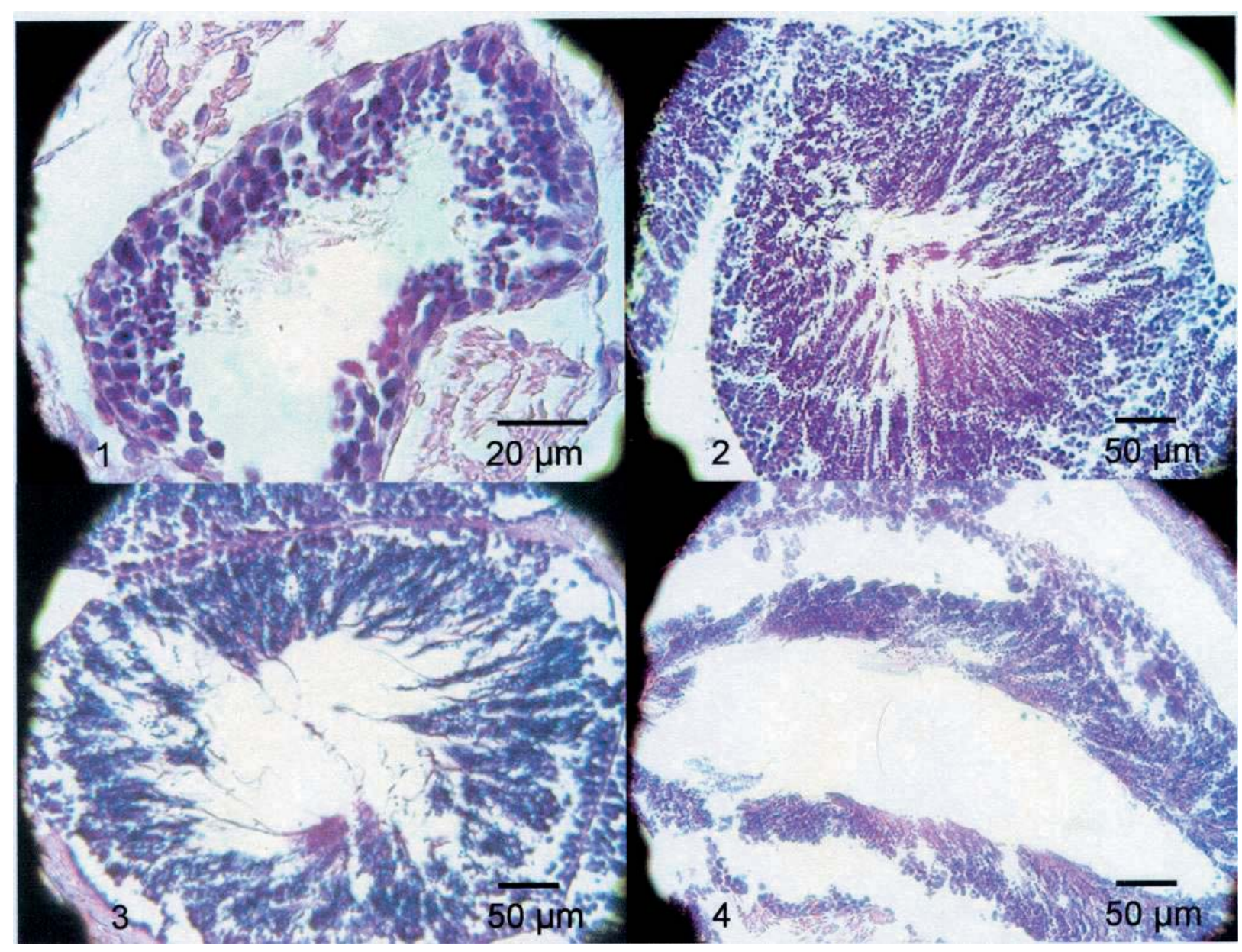

Figs. 1-4. Estágios do ciclo reprodutivo em machos de Donax hanleyanus: 1, início da maturação; 2, maturação máxima; 3, emissão parcial com proliferação; 4, emissão total.

forma original, e, em algumas ocasiões, a parede folicular aparece rompida. Concomitantemente, existem folículos em proliferação, sugerindo a retomada do ciclo reprodutivo (fig. 8).

A distribuição de freqüência dos estágios de maturação mostra que o ciclo reprodutivo na população é contínuo, pois tanto fêmeas quanto machos apresentaram os estágios de maturação máxima e/ou desova/emissão parcial de gametas em todos os meses do ano. $\mathrm{O}$ aumento crescente no percentual de indivíduos em emissão/desova total em dezembro de 2001, janeiro, fevereiro e abril de 2002, foi registrado após período de intensa atividade reprodutiva, de modo semelhante ao constatado para $D$. denticulatus Linné, 1758 por WADE (1968) nas praias da Jamaica, ainda que em D. hanleyanus o ciclo reprodutivo seja retomado quase que imediatamente.

A análise histológica das gônadas, associada às peculiaridades do estágio de desova/emissão total indica que, após a primeira maturação sexual, D. hanleyanus passa a se reproduzir, potencialmente, em todos os meses do ano, não havendo um período de repouso sexual absoluto.

O histograma de freqüência dos diversos estágios reprodutivos, para todos os espécimes adultos analisados (fig. 9), evidencia que indivíduos em início de maturação só não foram registrados em novembro de 2002, quando a totalidade dos espécimes analisados se encontrava em maturação máxima ou já estava em processo de emissão/ desova parcial, ainda que, neste último estágio, a proliferação de gametas ocorra concomitantemente.

A liberação parcial de gametas ocorre praticamente em todos os meses do ano, mas dois períodos estiveram marcados por uma intensificação na proliferação de gametas: outubro de 2001 e julho de 2002 (80\% e $85 \%$ dos espécimes analisados, respectivamente).

O primeiro pico de proliferação fez com que o percentual de indivíduos em desova/emissão parcial sofresse uma considerável elevação em novembro $(64,3 \%)$ e dezembro de 2001 (36,4\%) e em janeiro de $2002(28,0 \%)$. Em decorrência desse processo, os percentuais de indivíduos em estágio de desova/emissão total foram bastante elevados em janeiro (36\%) e fevereiro de 2002 $(45 \%)$. O evento voltou a se repetir em abril de 2002, quando 39\% dos indivíduos analisados apresentavam este estágio, após intensa desova/emissão total (62,5\%) ocorrida em março de 2002.

O segundo período de marcada atividade proliferativa (julho de 2002), também gerou elevado número de indivíduos em maturação máxima e em desova/ emissão parcial até dezembro de 2002, último mês de amostragem. 


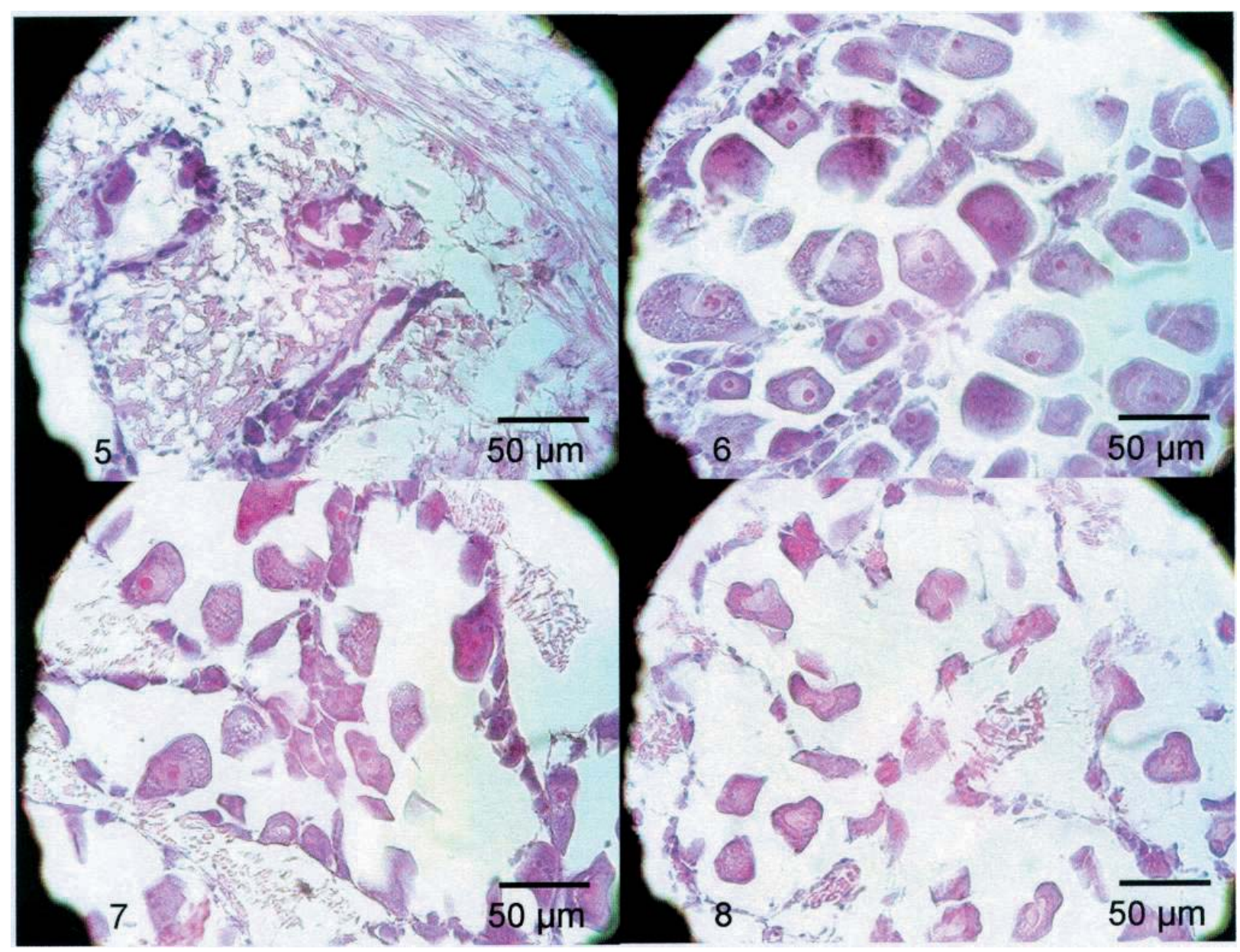

Figs. 5-8. Estágios do ciclo reprodutivo em fêmeas de Donax hanleyanus: 5, início da maturação; 6, maturação máxima; 7, desova parcial com proliferação; 8 , desova total.

Os resultados mostram que as variações naturais de salinidade não se configuram como um fator determinante para nenhum dos estágios do ciclo reprodutivo da população de $D$. hanleyanus em Arroio Teixeira (tab. I). Por outro lado, a temperatura da água parece exercer alguma influência, pelo menos no processo inicial de maturação e no estágio de desova/emissão parcial de gametas (fig. 10, tab. I).

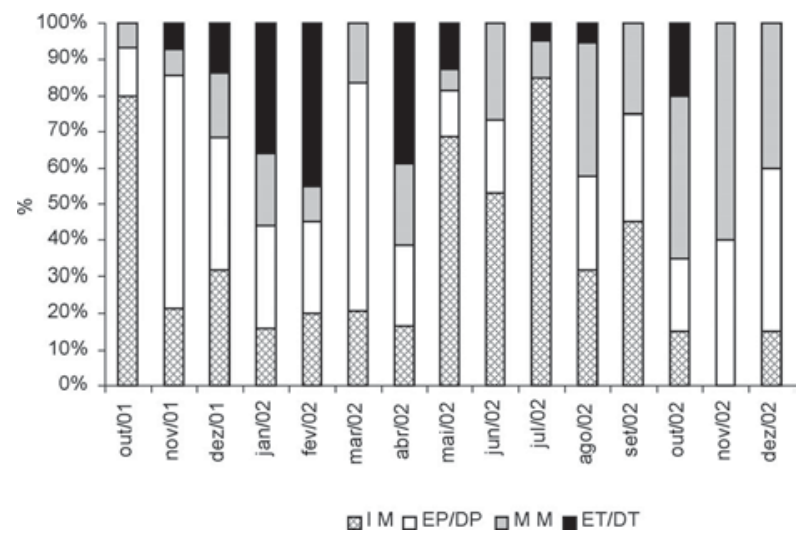

Fig. 9. Histograma mensal dos estágios reprodutivos em Donax hanleyanus (IM, início da maturação; EP/DP, emissão/desova parcial com proliferação; MM, maturação máxima; ET/DT, emissão/desova total)
Tabela I. Correlações (r) e nível de significância (p) entre a salinidade e a temperatura mensais da água e os percentuais de adultos de Donax hanleyanus por estágio reprodutivo (* , estatisticamente significativo).

\begin{tabular}{|c|c|c|c|c|}
\hline \multirow[t]{2}{*}{ Estágio reprodutivo } & \multicolumn{2}{|c|}{ temperatura } & \multicolumn{2}{|c|}{ salinidade } \\
\hline & $\mathrm{r}$ & $\mathrm{p}$ & $\mathrm{r}$ & $\mathrm{p}$ \\
\hline Início da $\mathrm{n}$ & $-0,556^{*}$ & 0,0 & 0,147 & 0,60 \\
\hline Emissão/Desova parcial & $0,578 *$ & 0,024 & $-0,300$ & 0,27 \\
\hline Maturação máxima & $-0,146$ & 0,603 & 0,000 & 0,9 \\
\hline Emissão/Desova Total & 0,410 & 0,129 & 0,093 & 0,74 \\
\hline
\end{tabular}

O início da maturação, com abundante proliferação de gametas, ocorreu com maior intensidade nos meses de outubro de 2001, e junho e julho de 2002, coincidindo com os meses em que a temperatura da água atingiu os menores valores $\left(18^{\circ} \mathrm{C}, 16^{\circ} \mathrm{C}\right.$ e $15^{\circ} \mathrm{C}$, respectivamente). No mês de maio, houve elevado percentual de animais em início de maturação $(57,89 \%)$ e a temperatura da água foi de $20^{\circ} \mathrm{C}$, relativamente quente para a área estudada e para a época do ano.

Já a elevação da temperatura da água parece estimular o processo de emissão/desova de gametas. Os maiores percentuais de animais neste estágio do ciclo reprodutivo ocorreram nos meses de verão, quando a temperatura da água variou entre $21^{\circ} \mathrm{C}$ e $26^{\circ} \mathrm{C}$.

O estágio de emissão/desova total atingiu os 

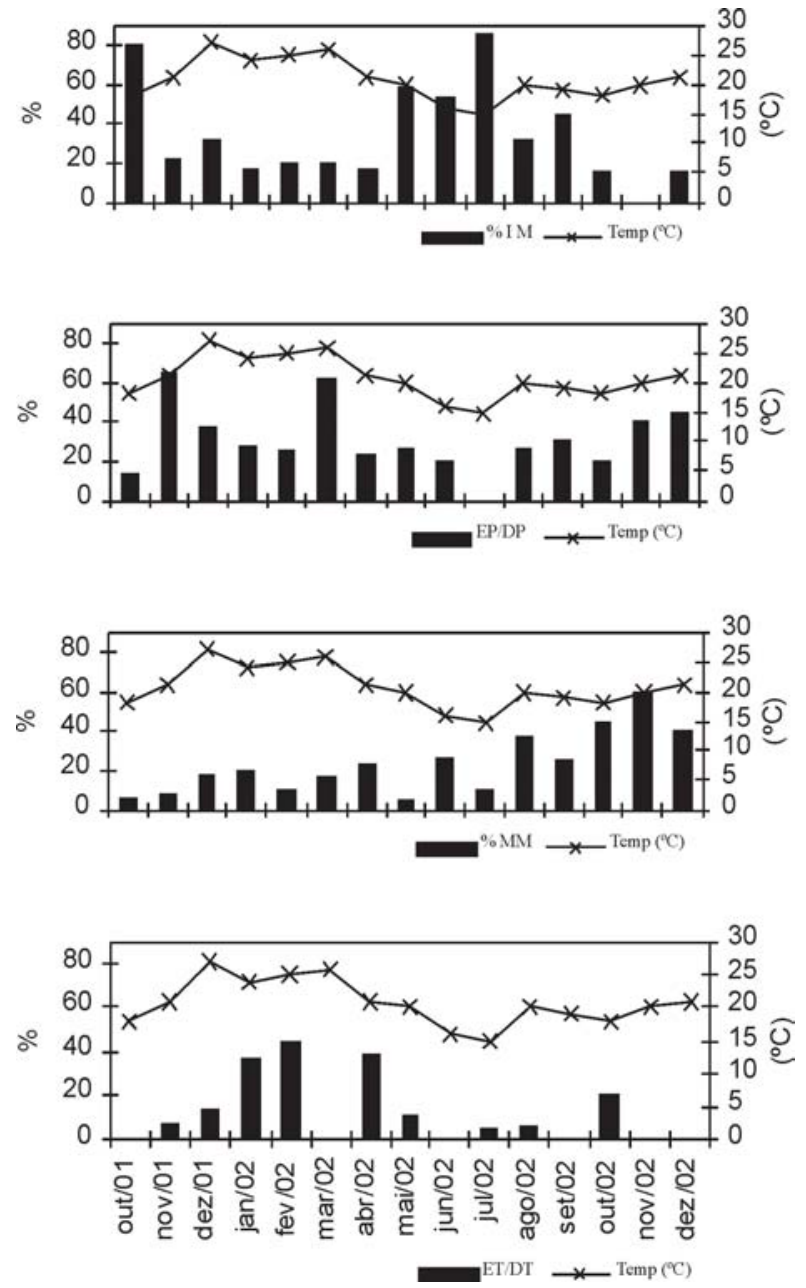

Fig. 10. Gráficos das temperaturas mensais da água e dos estágios reprodutivos em Donax hanleyanus (IM, início da maturação; EP/DP, emissão/desova parcial com proliferação; MM, maturação máxima; ET/DT, emissão/desova total).

maiores percentuais nos meses de janeiro, fevereiro e abril de 2002, refletindo um período de intensa atividade reprodutiva, justamente nos meses em que a temperatura da água do mar esteve mais elevada.

As flutuações sazonais de temperatura parecem estimular a atividade reprodutiva da espécie, pelo menos no que diz respeito ao início da maturação e à emissão/ desova de gametas.

\section{DISCUSSÃO}

A análise histológica demonstrou que a espécie é dióica, comprovando os estudos realizados com a espécie por Narchi (1974) e Penchaszadeh \& Olivier (1975), e os trabalhos de MouËZA \& Frenkiel-Renault (1973), Ansell (1983) e VÉLEZ (1985) sobre a inexistência de indivíduos hermafroditas em populações do gênero Donax.

A existência de diferentes estágios de maturação nos folículos gonadais de um mesmo animal é semelhante ao descrito por VéLEZ (1985) que, ao analisar as gônadas de $D$. denticulatus da Venezuela, encontrou diferentes estágios de maturação gonadal em um mesmo indivíduo.
A constatação de que $D$. hanleyanus apresenta esta mesma característica implicou em um aumento considerável no número de cortes histológicos analisados, em diferentes regiões das gônadas, a fím de determinar a condição de maturidade predominante nos folículos gonadais.

A ausência de um período de repouso sexual absoluto corrobora os resultados de PENCHASZADEH \& OLIVIER (1975) para as populações de D. hanleyanus da Argentina e diverge daqueles encontrados para as populações de D. trunculus Linné, 1758, de Portugal (GASPAR et al., 1999), Espanha (TIRAdo \& SAlAs, 1998) e Itália (ZEICHEN et al., 2002), que apresentam um estágio de inatividade gonadal prolongado.

O sucesso da tática reprodutiva apresentada pela população de $D$. hanleyanus na praia de Arroio Teixeira deve-se, principalmente, à capacidade de liberação parcial dos gametas durante todos os meses do ano, seja porque este é um mecanismo reprodutivo que permite aumentar o número de gametas produzidos durante o período de reprodução (VAZZOLER, 1996; GELAIN et al., 1999), seja porque amplia consideravelmente o período reprodutivo, aumentando a probabilidade de sucesso da prole.

AnSEll (1961) considerou a proliferação de gametas em Venus striatula (Da Costa, 1778) como uma resposta à disponibilidade de fitoplâncton, mais do que uma resposta à temperatura. No caso de $D$. hanleyanus, houve correlação negativa entre a temperatura da água e o percentual de animais em início de maturação, e correlação positiva entre a temperatura da água e a emissão/desova parcial de gametas, evidenciando a influência da temperatura da água sobre o ciclo reprodutivo da população.

Mesodesma mactroides Deshayes, 1854, espécie simpátrica à $D$. hanleyanus, também tem seu desenvolvimento gonadal termicamente controlado, com a desova ocorrendo quando a temperatura está em torno de $20^{\circ} \mathrm{C}$ (McLachlan et al., 1996).

A relação estabelecida por Ansell (1961) entre a abundância de fitoplâncton e a reprodução de $V$. striatula aparentemente não se aplica a $D$. hanleyanus, nem a $M$. mactroides, porque as praias arenosas do continente sulamericano exibem elevada produção primária (DEFEO \& SCARABINO, 1990), principalmente de diatomáceas como Asterionellopsis glacialis (Castracane) Round, 1990, Coscinodiscus sp. e Skeletonema costatum (Greville) Cleve 1873, alimentos preferenciais de D. hanleyanus. Esta alta produtividade primária, associada às características físicas desses ambientes, faz com que as zonas de praia e de arrebentação funcionem como um ecossistema semi-fechado (McLACHLAN, 1980), tornando a produção de fitoplâncton relativamente constante, condição que pode explicar a atividade reprodutiva de $D$. hanleyanus em todos os meses do ano.

Ainda que se possa inferir algum efeito da ação antrópica sobre a população estudada, principalmente durante os meses de verão, constatou-se que, no litoral norte do Rio Grande do Sul, D. hanleyanus encontra as condições ótimas para o desenvolvimento de seu ciclo de vida: a abundância e constância de fitoplâncton sustentam não só a sua sobrevivência, mas lhe garantem as condições permanentes de crescimento e reprodução. 
Com alimento abundante, ao longo de todo o ano, para sustentar o custo energético da reprodução, a temperatura da água agiria, então, como um fator interveniente apenas na intensidade da atividade reprodutiva desta população.

Agradecimentos. Ao CNPq e FAPERGS pelo apoio. À bolsista de Iniciação Científica, Fernanda Toscani, e à estagiária Carolina Heinz, pelo auxílio no processamento do material histológico. À Helena Sporleder Côrtes pela revisão ortográfica.

\section{REFERÊNCIAS BIBLIOGRÁFICAS}

Ansell, A. D. 1961. Reproduction, growth and mortality of Venus striatula (Da Costa) in Kames Bay, Millport. Journal of Marine Biology Association U.K., London, 41:191-215.

1983. The biology of the genus Donax. In: McLachlan, A. \& Erasmus, T. eds. Sandy beaches as ecosystems. Junk, The Hague. p.607-635.

Borzone, C. A.; Vargas, K. M. et al. 2001. Aspectos da reprodução e dinâmica populacional de Chione pubera (Bory Saint-Vicent) (Bivalvia, Veneridae) no sul do Brasil. Revista Brasileira de Zoologia, Curitiba, 18(2):333349 .

Cardoso, R. S. \& Veloso, V. G. 2003. Population dynamics and secondary production of the wedge clam Donax hanleyanus (Bivalvia: Donacidae) on a high-energy, subtropical beach of Brasil. Marine Biology, Heidelberg, 142: $153-162$.

Coe, W. R. 1956. Fluctuations in populations of littoral marine invertebrates. Journal of Marine Research, New Haven, 15(1-3):212-232.

Defeo, O. \& Alava, A. 1995. Effects of human activies on long-term trends in sandy beach populations: the wedge clam Donax hanleyanus in Uruguay. Marine Ecological Progress Series, Oldendorf, 123:73-82.

Defeo, O. \& Scarabino, V. 1990. Ecological significance of a possible deposit-feeding strategy in Mesodesma mactroides (Deshayes, 1854) (Mollusca: Pelecypoda). Atlántica, Santiago, 12:55-65.

Gaspar, M. B.; Ferreira, R. \& Monteiro, C. C. 1999. Growth and reproductive cycle of Donax trunculus L. (Mollusca: Bivalvia) off Faro, southern Portugal. Fisheries Research, Aberdeen, 41:309-316.

Gelain, D.; Fialho, C. B. \& Malabarba, L. R. 1999. Biologia reprodutiva de Serrapinnus calliurus (Boulenger, 1900) (Characidae, Cheirodontinae) do Arroio do Ribeiro, Barra do Ribeiro, Rio Grande do Sul, Brasil. Comunicações do Museu de Ciências e Tecnologia da PUCRS, Sér. Zoologia, Porto Alegre, 12:71-82.

GiL, G. M. \& Thомé, J. W. 2001. Influência de variáveis ambientais sobre uma população de Donax hanleyanus Philippi, 1847 (Mollusca, Bivalvia, Donacidae). Biotemas, Florianópolis, 14:109-126.
McLachlan, A. 1980. Exposed sandy beaches as semi-closed ecosystems. Marine Environmental Research, Little River, 4:59-63.

McLachlan, A.; Dugan, J. E. et al. 1996. Beach clam fisheries. Oceanography and Marine Biology: an Annual Review, London, 34:163-232.

McLachlan, A. \& Hanekon, N. 1979. Aspects of biology, ecology and seasonal fluctuations and biochemical composition of Donax serra in the East Cape. South African Journal of Zoology, Pretoria, 14:183-193.

Mclachlan, A.; Wooldridge, T. \& Dye, A. H. 1981. The ecology of sandy beaches in southern Africa. South African Journal of Zoology, Pretoria, 16:219-231.

Mesquita, E. F. M.; Abreu, M. G. \& Lima, F. C. 2001. Aspectos gametogênicos de Iphigenia brasiliana (Lamarck) (Bivalvia, Donacidae) da Lagoa de Itaipu, Niterói, Rio de Janeiro, Brasil. Revista Brasileira de Zoologia, Curitiba, 18(2): $523-528$

Morrison, J. P. E. 1971. Western Atlantic Donax. Proceedings of the Biological Society of Washington, Washington, 83(48):545-568.

Mouëza, M. \& Frenkiel-Renault, L. 1973. Contribution à l'étude de la biologie de Donax trunculus L. (Mollusques Lamellibranches) dans 1'Algèrois: la reproduction. Cahiers de Biologie Marine, Paris, 14:261-283.

Narchi, W. 1974. Aspectos ecológicos e adaptativos de alguns bivalves do litoral paulista. Papéis Avulsos de Zoologia, São Paulo, 27(19):235-262.

1976. Ciclo anual da gametogênese de Anomalocardia brasiliana (Gmelin, 1791) (Mollusca Bivalvia). Boletim de Zoologia da Universidade de São Paulo, São Paulo, 1:331-350.

Penchaszadeh, P. E. \& Olivier, S. R. 1975. Ecología de una población de "berberecho" (Donax hanleyanus) en Villa Gesell, Argentina. Malacologia, Ann Arbor, 15(1):133146 .

Sicardi, O. E. 1993. Los Donacidae actuales. Comunicaciones de la Sociedad Malacológica del Uruguay, Montevideo, 7:325-337.

Tirado, C. \& Salas, C. 1998. Reproduction and fecundity of Donax trunculus L. 1758 (Bivalvia: Donacidae) in the littoral of Málaga (southern Spain). Journal of Shellfisheries Research, Miami, 17:169-176.

VAzzoler, A. E. A. M. 1996. Biologia da reprodução de peixes teleósteos: teoria e prática. Maringá, EDUEM. $169 \mathrm{p}$.

VÉLEZ, A. 1985. Reproductive biology of the tropical clam Donax denticulatus in eastern Venezuela. Caribbean Journal of Science, Mayagüez, 21(3,4):125-136.

WADE, B. A. 1968. Studies on the biology of the West Indian beach clam, Donax denticulatus Linné. 2. Life history. Bulletin of Marine Science, Miami, 18:876-901.

Zeichen, M. M.; Agnesi, S. et al. 2002. Biology and population dynamics of Donax trunculus L. (Bivalvia: Donacidae) in south Adriatic coast. Estuarine, Coastal and Shelf Science, Stirling, 54(6):971-982. 